\section{Social involvement, food safety and food security}

Gaetano Liuzzo, ${ }^{1}$ Stefano Bentley, ${ }^{2}$ Federica Giacometti, ${ }^{3}$ Silvia Piva, ${ }^{3}$ Andrea Serraino ${ }^{3}$

${ }^{1}$ Modena Local Health Unit, Carpi; ${ }^{2}$ Department of Veterinary Sciences, University of Parma; ${ }^{3}$ Department of Veterinary Medical Sciences, University of Bologna, Ozzano dell'Emilia, Italy

\begin{abstract}
The paper describes the terminology of risk assessment in the view of food safety: different aspects of social involvement are defined and discussed; the terms Document, Expert, Risk manager, Lay Knowledge, Participant, Participation, Citizens' involvement, Community of interest, Consultation, Trust and Social trust are presented. Also, the terms Adverse effects, Human Illness Source Attribution, Food hygiene, Emerging disease, Safety, Food security and Food safety are discussed.
\end{abstract}

\section{English version}

\section{Document}

Legal definition: any content whatever its medium (written on paper or stored in electronic form or as a sound, visual or audiovisual recording) concerning a matter relating to the policies, activities and decisions falling within the institution's sphere of responsibility (European Commission, 2001).

Therefore, the subject of the right of access is not the document itself but the information contained therein.

\section{Expert}

A person whose competence to carry out expertise work is formally recognized by an enabling process. His main task is the technical risk assessment and the identification of solutions, but he should also provide full understanding of risks and the reasons of the proposed solutions (Biocca, 2002).

\section{Risk manager}

Representative of a regional/national government or international governmental organization with responsibility for microbiological risk management (MRM).

The definition of Risk Manager stems from the definition for risk management which does not include all of the individuals who are involved in the implementation phase and related activities associated with MRM, i.e., MRM decisions are largely implemented by industry and other interested parties. The focus of the definition on risk manager is restricted to governmental organizations with authority to decide on the acceptability of risk levels associated to foodborne hazards (CAC, 2007).

\section{Lay knowledge}

The knowledge of non-experts, of ordinary people (Bucchi, 2002).

\section{Participant}

A person, group or body that has interests in play, its solutions, and the general public participation process (Santé Canada, 2000).

\section{Participation}

A series of activities and relationships that include two-way communication, public education, consultation of participants, citizen involvement, advisory bodies, partnerships, and the power of joint decisionmaking. It includes continuous exchanges between participants who agree to mutually influence on decisions to be taken (Santé Canada, 2000).

\section{Citizens' involvement}

The process of informed dialogue and deliberation among citizens, usually involves the exchange of ideas and values, the possibility of mutual learning, the decision making on the best ways to proceed, as well as the creation of partnerships to give solutions (Santé Canada, 2000).

\section{Community of interest}

A group of participants gathering the authors of a question, opinion or common concern often for a limited period of time (Santé Canada, 2000).

\section{Consultation}

The request to express views from participants in the context of developing public health policies, often through the creation of forums, roundtables or consultative councils. Consultation is part of the participation process but it is not its synonymous (Santé Canada, 2000).

\section{Trust}

It is the belief that most of the future events will be realized as expected (Siegrist et al., 2003).

According to the most accredited theories, trust is based on three factors: the perception that the other party is benevolent (doing things for my good), the perception that the other party is competent (i.e., doing the best things), the perception that both parties share the same values (e.g. stealing is bad, killing is a sin). When a food scandal breaks out, trying to prove one's innocence
Correspondence: Andrea Serraino, Department of Veterinary Medical Sciences University of Bologna, via Tolara di Sopra 50, 40064 Ozzano dell’Emilia (BO), Italy.

Tel.: +39.051.2097323.

E-mail: andrea.serraino@unibo.it

Key words: Social involvement, adverse effects, food safety, food security.

Contributions: The authors contributed equally.

Conflict of interest: The authors declare no potential conflict of interest.

Received for publication: 2 March 2018.

Accepted for publication: 13 March 2018.

This work is licensed under a Creative Commons Attribution-NonCommercial 4.0 International License (CC BY-NC 4.0).

(C) Copyright G. Liuzzo et al., 2018

Licensee PAGEPress, Italy

Italian Journal of Food Safety 2018; 7:7394

doi:10.4081/ijfs.2018.7394

is not as important as having first built a common ground of shared values. And if this hasn't been done previously, it's necessary to promote a communication that emphasizes how the company and the community have the same values (Savadori and Rumiati, 2005).

\section{Social trust}

Propensity to trust who has the responsibility to make decisions and actions related to the management of technology, the environment, medicine or other areas of public health and safety (Siegrist and Cvetkovich, 2000).

\section{Adverse effects}

Changes in morphology, physiology growth, evolution or lifespan of an organism that result in an impairment of functional capacity or ability to compensate for additional stress or increase sensitivity to the nuisance effects of other environmental influences.

The adverse health effects include diseases whose duration/severity can be variable, easy to treat as well as requiring hospitalization or even leading to chronic illnesses with potentially fatal outcome. At a population level, the appearance of asymptomatic pathogens' carriers can be classified as a health hazard, as it can lead to multiplication, excretion and transmission of the pathogen to other individuals who may thus develop a secondary transmission disease or die. Decisions to determine whether an effect is adverse or not must be based on a expert judgement (FAO/OMS, 2004; 2009). 


\section{Human illness source attribution}

Human illness source attribution may be defined as the partitioning of the human disease burden of one or more foodborne infections to specific sources, e.g. enteric pathogens. (Batz et al., 2005; EFSA, 2008; Pires et al., 2009).

The term source in this definition and context indicates where the pathogen that caused the disease is located (Pires et al., 2009), and what its reservoir is (any biological and/or non-biological system in which the pathogen normally lives and multiplies) (Last, 1995). The importance of attributing the source of human food-borne diseases is crucial to assess the effectiveness of risk management interventions and the relationship between food and pathogens along the food chain, from production to consumption (Batz et al., 2005). Basic prerequisite for any type of method used for the attribution is the classification of foods (or categorization) and the stage of the chain where the attribution can take place, including the path from production to consumption.

There are several approaches and information sources used for this purpose; according to a scheme proposed by several authors (Batz et al., 2005; Pires et al., 2009) two major approaches (designated as epidemiological and microbiological) have to be considered, plus a third one, which is applied in the use of the Expert Judgment. The epidemiological approach is based on surveillance systems implemented in public health while the microbiological approach relies on human, animal and food monitoring plans.

Recently, Pires et al. (2009), coded and reviewed the various methods for attributing human illnesses to specific sources, completing the work begun by Batz et al. (2005). Hence the following classification:

1. Microbiological Approach:

Microbial subtyping

Comparative Exposure Assessment

(Risk Assessment)

2. Epidemiological approach:

Analysis of data from outbreak

investigations

Study of sporadic cases

Case-control study

Cohort studies

Case-series studies

The microbial subtyping approach is based on the characterization of isolates of a specific pathogen (e.g. Salmonella) in order to compare the subtypes of isolates from different sources (e.g., animals, food) with the human isolates. The application of this approach assumes that there is a clear predominance of subtypes for each animal reservoir and hence the detection of human disease of this subtype linked to that source.

\section{Food hygiene}

All conditions and measures necessary to ensure the safety and suitability of food at all stages of the food chain (CAC, 2004).

\section{Emerging disease}

The concept of emerging disease may correspond to the development of a new disease (implying a new mechanism of pathogenicity, a new pathogen, a new vector, a new strain, adaptation to a new species or the appearance of a genetic anomaly) different from the already described and known pathological entity (in terms of severity and/or symptoms and / or epidemiological dynamics); but may also be a previously known disease, but whose incidence, measured over a reference period of e.g. 20 years, has increased significantly in a defined area or population (Barnouin and Vourch, 2004).

\section{Safety}

Acceptable level of risk; a subjective measure of the acceptability of risk. In literature, this term is generally used when discussing safety for human health. (Ahl et al., 1993).

\section{Food security}

The first available definition of food security can be traced back to the Rome Declaration on World Food Security which states: ...Food security exists when all people, at all times, have physical and economic access to sufficient, safe and nutritious food to meet their dietary needs and food preferences for an active and healthy life... (FAO, 1996). With the term food security, we refer specifically to developing countries, and hence food availability (Martel, 1998).

\section{Food safety}

The definition of food safety appears in the Codex Document General Principles of Food Hygiene as follows: Food safety assurance that food will not cause harm to the consumer when it is prepared and/or eaten according to its intended use.

The term food safety, openly refers to the biological and chemical safety, thus meaning the absence of pathogenic substances or their presence in non-hazardous quantities (Babuscio, 2005; CAC/RCP, 1969; CAC, 2004; Martel, 1998).

\section{Italian version}

\section{Documento}

[definizione legale: «documento», qualsiasi contenuto informativo, a prescindere dal suo supporto (testo su supporto cartaceo o elettronico, registrazione sonora, visiva o audiovisiva) che verta su aspetti relativi alle politiche, iniziative e decisioni di competenza dell'istituzione (European Commission, 2001).

Oggetto quindi del diritto di accesso non è il documento in sé, bensì le informazioni in esso contenute.

\section{Esperto}

Una persona la cui competenza a realizzare lavori di expertise è formalmente riconosciuta da un processo di abilitazione. Il suo compito principale è la stima tecnica del rischio e l'identificazione di soluzioni, ma fra le sue funzioni dovrebbe esserci anche quella di far comprendere i rischi e le ragioni delle soluzioni proposte (Biocca, 2002).

\section{Risk manager}

Rappresentante di un governo regionale/nazionale o di un'organizzazione governativa internazionale responsabile della gestione dei rischi microbiologici (MRM).

La definizione di Risk Manager deriva dalla definizione di gestione del rischio che non include tutti gli individui che sono coinvolti nella fase di implementazione e le attività correlate associate a MRM, cioè le decisioni MRM sono ampiamente implementate dall'industria e da altre parti interessate. L'obiettivo della definizione sul Risk Manager è limitato alle organizzazioni governative autorizzate a decidere sull'accettabilità dei livelli di rischio associati ai pericoli alimentari (CAC, 2007).

\section{Sapere laico}

Il sapere dei non esperti, della gente comune (Bucchi, 2002).

\section{Partecipante}

Una persona, un gruppo od un organismo che ha interessi in gioco, nelle sue soluzioni e nel processo generale di partecipazione del pubblico (Santé Canada, 2000).

\section{Partecipazione}

Una serie di attività e di relazioni che comprendono la comunicazione nei due sensi, l'educazione del pubblico, la consultazione degli intervenuti, il coinvolgimento dei cittadini, gli organismi consultivi, i paternariati, il potere di decisione congiunto. Comprende gli scambi continui fra partecipanti che accettano di influenzarsi mutuamente sulle decisioni da prendere (Santé Canada, 2000).

\section{Coinvolgimento dei cittadini}

Processo di dialogo informato e di deliberazione fra i cittadini, comprende abitualmente lo scambio di idee e di valori, di possibilità d'apprendere gli uni da gli altri, di prese di decisione sui migliori modi di procedere, così come la creazione di paterna- 
riati per dare vita a delle soluzioni (Santé Canada, 2000).

\section{Comunità di interesse}

Gruppo di partecipanti che riunisce gli autori di una questione, di una opinione o di una preoccupazione comune, speso per un periodo di tempo limitato (Santé Canada, 2000).

\section{Consultazione}

La sollecitazione ad esprimere punti di vista da parte dei partecipanti nel contesto del processo di elaborazione di politiche di sanità pubblica, spesso attraverso la realizzazione di forum, tavole rotonde o consigli consultativi. La consultazione è parte del processo di partecipazione ma non né è sinonimo (Santé Canada, 2000).

\section{Fiducia}

È la convinzione che la maggior parte degli eventi futuri si realizzi come atteso (Siegris et al., 2003). Secondo le teorie più accreditate tre sono i fattori che stanno alla base della fiducia: la percezione che l'altra parte sia benevola (ovvero faccia le cose per il mio bene), la percezione che l'altra parte sia competente (ovvero, faccia le cose migliori), la percezione che io e l'altra parte abbiamo dei valori simili (es. rubare è male, uccidere è peccato). Quando esplode uno scandalo alimentare, non conta tanto dimostrare con dati e fatti alla mano la propria innocenza, quanto piuttosto l'avere costruito prima un terreno di valori condivisi. E se non lo si è fatto prima, è necessario e importante promuovere una comunicazione che enfatizzi come l'azienda e le persone abbiano gli stessi valori (Savadori e Rumiati, 2005).

\section{Fiducia sociale}

La disponibilità a fidarsi di chi ha responsabilità nella presa di decisioni e azioni relative alla gestione della tecnologia, dell'ambiente, della medicina o altri settori della salute pubblica e della sicurezza (Siegrist e Cvetkovich 2000).

\section{Effetti nocivi}

Cambiamenti nella morfologia, nella fisiologia, nella crescita, nell'evoluzione o nella durata della vita di un organismo che si traducono in una alterazione della capacità funzionale o di una capacità che compensi uno stress supplementare o da un aumento della sensibilità agli effetti nefasti di altre influenze ambientali. Gli effetti nocivi sulla salute comprendono malattie di gravità (morbilità) e durata variabile, malattie risolutive leggere come malattie che necessitano di ospedalizzazione od ancora che conducono a patologie croniche fino alla morte (mortalità). A livello di popolazione l'apparizione di portatori asintomatici del patogeno può essere classificata come effetto nocivo sulla salute in quanto può condurre alla moltiplicazione, all'escrezione e alla trasmissione dell'organismo patogeno ad altri individui che possono così sviluppare una malattia o morire (trasmissione secondaria). Le decisioni volte a stabilire se un effetto è o non è avverso devono essere basati su di un giudizio d'esperti (FAO/OMS, 2004; 2009).

\section{Attribuzione della fonte delle malat- tie umane}

Letteralmente: Attribuzione della fonte delle malattie umane è, nel campo della Sicurezza degli Alimenti, quel processo che consente di quantificare la quota, rispetto al carico di malattie umane (Burden of disease) dovute a patogeni enterici, attribuibile agli alimenti e conoscere quali sono gli alimenti che contribuiscono a formare questa quota (Batz et al., 2005; EFSA, 2008; Pires, et al., 2009).

Il termine source in questa definizione ed in questo contesto indica dove il patogeno che ha causato la malattia si trova (Pires et al., 2009), quindi in via definitiva quale è il suo reservoir (serbatoio), intendendo per questo qualsiasi sistema biologico e/o non biologico in cui il patogeno normalmente vive e si moltiplica (Last, 1995). L'importanza di attribuire la fonte della malattie umane di origine alimentare è fondamentale soprattutto per poter valutare l'effettiva efficacia degli interventi di gestione del rischio ed i rapporti fra alimento e patogeno lungo la filiera alimentare, dalla produzione al consumo (Batz et al., 2005). Presupposto fondamentale per qualsiasi tipo di metodo utilizzato per l'attribuzione della fonte è la classificazione degli alimenti (o categorizzazione) nonché il punto della filiera dove l'attribuzione può avvenire, includendo quindi il percorso che dalla produzione arriva al consumo. Sono diversi gli approcci proposti e soprattutto le sorgenti d'informazioni utilizzate a questo scopo; secondo uno schema condiviso da più autori (Batz et al., 2005; Pires et al., 2009) si considerano 2 grandi categorie designate come: epidemiologiche e microbiologiche. A questi si aggiunge un terzo approccio che si concretizza nel ricorso al Giudizio degli Esperti (Expert Judgment). L'approccio epidemiologico si basa sui sistemi di sorveglianza messi in atto in sanità pubblica mentre l'approccio microbiologico fa affidamento ai piani di monitoraggio in campo umano, animale e alimentare. Recentemente Pires et al. (2009), hanno codificato e passato in rassegna le varie metodologie per l'attribuzione delle malattie umane a specifiche fonti completando il lavoro iniziato da Batz et al. (2005). Ne deriva la seguente classificazione:

1. Approccio Microbiologico: Subtipizzazione microbica Valutazione comparativa dell'esposizione (valutazione del rischio)

2. Approccio epidemiologico: Analisi dei dati generati da investigazioni realizzate nel corso di focolai

Studio di casi sporadici

Studio caso-controllo

Studi di coorte

Studi di serie di casi

Nell'Approccio Microbiologico mediante subtipizzazione il presupposto fondamentale è l'isolamento del patogeno dalle varie fonti (sorgenti) e nei casi umani di malattia. Per esempio, la Salmonella isolata da fonti animali e/o alimentari viene subtipizzata e confrontata in modo quantitativo con la Salmonella isolata e subtipizzata nel corso di malattia umana. L'assunto riguarda il fatto che vi è una predominanza distintiva di sottotipi per ogni reservoir animale e quindi l'individuazione in corso di malattia umana di questo sottotipo viene assunto come originato da quella fonte.

\section{Igiene alimentare}

Insieme delle condizioni e delle misure necessarie per assicurare la sicurezza e la salubrità degli alimenti in tutte le tappe della catena alimentare (CAC, 2004).

\section{Malattia emergente}

Il concetto di malattia emergente può corrispondere: allo sviluppo di una nuova malattia (sottintendendo un nuovo meccanismo di patogenicità, un nuovo patogeno, un nuovo vettore, un nuovo ceppo, l'adattamento ad una nuova specie o l'apparizione di una anomalia genetica) distinta (per la sua gravità e/o i suoi sintomi e/o la sua dinamica epidemiologica) dall'entità patologica già descritta e conosciuta; ad una malattia a priori conosciuta, ma la cui incidenza, misurata su un periodo di riferimento per es. di 20 anni, è aumentato significatamente al livello di una zona o di una popolazione definita (Barnouin e Vourch, 2004),

\section{Salubrità degli alimenti}

Assicurazione che gli alimenti siano accettabili per il consumo umano conformemente all'uso al quale sono destinati (CAC, 2004).

\section{Sicurezza}

Livello di rischio giudicato accettabile, decisione soggettiva sull'accettabilità di un rischio. Nella letteratura, il concetto è abitualmente utilizzato nella discussione sulla sicurezza per la salute umana (Ahl et al., 1993). 


\section{Sicurezza alimentare}

La prima definizione rinvenibile di sicurezza alimentare è quella contenuta nel Rome Declaration on World Food Security che così recita: ...La sicurezza alimentare esiste quando tutte le persone, in ogni momento, hanno accesso fisico ed economico a cibi sufficienti, sicuri e nutrienti per soddisfare le loro esigenze alimentari e le preferenze alimentari per una vita attiva e sana ... (FAO, 1996). Con il termine di Sicurezza Alimentare (Food Security) ci riferiamo specificatamente e soprattutto ai paesi in via di sviluppo e quindi agli aspetti di disponibilità degli alimenti (Martel, 1998).

\section{Sicurezza degli alimenti}

La definizione di sicurezza alimentare nel senso di Food Safety compare nella norma del Codex General principles of food hygiene nel seguente modo: Sicurezza alimentare - garanzia che gli alimenti non causino danni al consumatore quando sono preparati elo consumati secondo il suo uso previsto. La sicurezza alimentare è quindi la garanzia che l'alimento non sia causa di danno per il consumatore, alla condizione che il consumatore lo prepari e lo consumi conformemente all'uso al quale è destinato. È interessante notare come nella versione francese risulti ancor più evidente il passaggio del concetto di alimento come sicurezza a quello di sicurezza dell'alimento (Babuscio, 2005), Food safety viene infatti tradotto come Sécurité des aliments, la sicurezza non è alimentare ma dell'alimento.

Assicurazione che gli alimenti sono senza pericolo per il consumatore quando sono preparati e/o consumati conformemente all'uso al quale sono destinati (CAC, 2004). Con il termine di sicurezza degli alimenti ci si riferisce esplicitamente alla sicurezza chimico-biologica degli alimenti nel senso dell'assenza di sostanze patogene o nella loro presenza in quantità non pericolo- se, include quindi una valenza igienica e corrisponde al termine inglese Food Safety.

\section{References}

Ahl AS, Acree JA, Gipson PS, McDowell RM, Miller L, McElvaine MD, 1993. Standardisation of nomenclature for animal health risk analysis. Revue Scientifique et tecnique Office International des Epizooties, 12:104553.

Babuscio T. (2005). Alimenti Sicuri e Diritto. Analisi di problemi giuridici nei sistemi amministrativi delle Autorità per la Sicurezza Alimentare Europee e Statunitense. Istituto di Diritto Agrario Internazionale e Comparato Firenze, Giuffrè Editore, Milano.

Batz MB, Doyle MP, Morris GJ, Painter J, Singh R, Tauxe RV, Taylor MR, Lo Fo Wong DMA, 2005. Attributing Illness to Food, Emerging Infectious Diseases 11:993-9.

Biocca M., 2002. La comunicazione sul rischio per la salute nel teatro di Sagredo, Centro Scientifico Editore, Torino.

Bucchi M. Scienza e società, Il Mulino editore, Bologna, 2002.

CAC, 2004. Textes de base concernant l'hygiene alimentaire. $12^{\mathrm{a}}$ edition, 2004.

CAC/RCP, 1969. General principles of food hygiene1-1969.

EFSA, 2008. Overview of methods for source attribution for human illness from foodborne microbiological hazards. EFSA J 764:1-43.

European Commission, 2002. Regulation of the European Parliament and of the Council of 28 January 2002 laying down the general principles and requirements of food law, establishing the European Food Safety Authority and laying down procedures in matters of food safety, 178/2002/CE. In:
Official Journal, L 31/1, 01/02/2002.

European Commission. Regulation (European Commission) No 1049/2001 of The European Parliament and of the Council of 30 May 2001 regarding public access to European Parliament, Council and Commission documents. In Official Journal of the European Communities, L 145/43.

FAO, Rome Declaration on World Food Security, 1996. Word Food Summit, Rome, 13-17 November 1996.

FAO/OMS, 2004. Caractérisation des dangers liés à la présence des pathogènes dans les aliments et dans l'eau, Directives, Série Évaluation des risques microbiologiques $n^{\circ} 3$. 1-63.

FAO/OMS, 2009. Caractérisation des risques liés aux dangers microbiologiques d'origine alimentaire, Directives, Série évaluation des risques microbiologiques 17, Rome, 2009.

Last JM, 1995. A dictionary of epidemiology, Third ed. New York: Oxford University Press Editor.

Martel P, 1998. La sécurité des aliments, INRA.

Pyres SM, Evers EG, Van Pelt W, Ayers T, Scallan E, Angulo FJ, Havelaar A, Hald T, Med-Vet-Net Workpackage 28 Working Group, 2009. Attributing the Human Disease Burden of Foodborne Infections to Specific Sources. Foodborne Pathog Dis 6:417-24.

Salvadori L, Rumiati R, 2005. Nuovi rischi, vecchie paure, Il Mulino Ed.

Santé Canada, 2000. Participation du public: cadre et lignes directrices.

Siegrist M, Cvetkovich G, 2000. Perception of hazards: The role of social trust and knowledge. Risk Anal 20:713-9.

Siegrist M, Earle TC, Gutscher H, 2003. Test of a trust and confidence model in the applied context of electromagnetic field (EMF) Risk. Risk Anal 23;705-16. 